\title{
Experiments and Simulations of Hyperthermal Xe Interacting with an Ordered 1-Decanethiol/Au(111) Monolayer: Penetration Followed by High-Energy, Directed Ejection ${ }^{\dagger}$
}

\author{
K. D. Gibson, N. Isa, and S. J. Sibener* \\ The James Franck Institute and Department of Chemistry, The University of Chicago, 5640 South Ellis Avenue, \\ Chicago, Illinois 60637
}

Received: September 13, 2005; In Final Form: October 27, 2005

\begin{abstract}
A study of the interaction of hyperthermal Xe with a well-ordered standing-up phase of 1-decanethiol adsorbed on $\mathrm{Au}(111)$ is presented. Experimentally, double-differential measurements were made of the postcollision Xe kinetic energy as a function of incident and final angles. These experiments are compared to classical trajectory calculations. The results show the two expected channels: direct-inelastic scattering from the surface and accommodated Xe due to trapping-desorption. There is also evidence of a further interaction mechanism. This involves the penetration of the atom deep into the channels between the aligned chains of the monolayer. When the collision energy has been dissipated, the implanted Xe is expelled as the chains return to their equilibrium positions. The expelled Xe leaves the surface with an energy much higher than expected for trapping-desorption, and with an angular-intensity distribution peaked close to the direction of the 1-decanethiol chain orientation. For this reason, we call this new scattering mechanism directed ejection.
\end{abstract}

\section{Introduction}

This paper presents experimental and theoretical results for $\mathrm{Xe}$, with a kinetic energy of $1.5-3.6 \mathrm{eV}$, colliding with an ordered, standing phase of 1-decanethiol adsorbed on $\mathrm{Au}(111)$. These results are an extension of previous results for the lighter rare gases $\mathrm{Ne}^{1,2}$ and $\mathrm{Ar}^{3}$ In those studies, the time-of-flight (TOF) spectra of the in-plane scattering as a function of incident and final angle were measured. The double-differential measurements show two distinct features with different average energies. For Ne with an incident energy, $E_{\mathrm{i}}$, of $19-550 \mathrm{meV}$, the faster was attributed to direct-inelastic scattering, and the second to almost, but not quite, thermalized Ne. For Ar with $E_{\mathrm{i}}=65-$ $650 \mathrm{meV}$, the faster feature was also attributed to direct-inelastic scattering, the slower was due to trapping-desorption, with an average energy appropriate to the surface temperature, $T_{\mathrm{s}}$, and a cosine $\left(\Theta_{\mathrm{f}}\right)$ angular-intensity distribution. In both $\mathrm{Ne}$ and $\mathrm{Ar}$ experiments, the atom that scattered without thermalizing with the surface occurred at glancing incident angles and, for a given incident angle, at glancing final angles. According to our classical scattering calculations, the colliding $\mathrm{Ne}$ and $\mathrm{Ar}$ atoms greatly distort the surface of the SAM at impact but rarely penetrate below the level of the terminal $\mathrm{CH}_{3}$.

The scattering of high-energy Xe from various surfaces has been extensively studied, for example, the solids $\mathrm{Ni}(111),{ }^{4}$ $\mathrm{Pt}(111),{ }^{5-7}$ and $\mathrm{GaAs}(110),{ }^{8,9}$ as well as organic liquids ${ }^{10,11}$ and molten metals. ${ }^{12}$ The details are beyond the scope of this introduction, but the important point is that the scattering is attributed purely to interaction of the Xe with the surface. There is no penetration. For $1 \mathrm{eV}$ Ar impinging upon $180 \mathrm{~K}$ ice, there was calculated to be penetration of the rare gas, but only after adsorption on the surface. ${ }^{13}$

\footnotetext{
† Part of the special issue "William Hase Festschrift".

* To whom correspondence should be addressed. E-mail: s-sibener@uchicago.edu.
}

The structure of the 1-decanethiol monolayer is unique relative to the other surfaces. It is hexagonally ordered, and consists of parallel standing-up chains, tilted from normal by $34^{\circ}$, with relatively weak interchain forces. This results in an array of channels between each of the chains. In this paper, evidence will be presented to show that hyperthermal atoms can actually penetrate far into these channels. The energy of the incoming projectile is quickly dissipated during the penetrating trajectory. The atom is then expelled, without damaging the monolayer, as the chains return to their equilibrium positions. The atom leaves the surface at energies much greater than expected for accommodation and in a direction preferentially along the chain tilt relative to the $\mathrm{Au}(111)$ substrate. This is a mechanism distinct from the direct-inelastic and trappingdesorption surface scattering of the previous systems. Day et al. ${ }^{14,15}$ predict that when a $0.82 \mathrm{meV}$ Ar atom impinges on an alkanethiol monolayer, they will deeply penetrate and be ejected along the chain tilt direction with greater than thermal energy when the chains are far enough apart. This supports the results of our experimental and theoretical studies, described in this paper, and presented at earlier symposia. ${ }^{16,17}$

\section{Experiment and Trajectory Calculations}

The experimental apparatus and methods have been covered elsewhere, ${ }^{1,18}$ so only a brief description will be presented. The experiments were carried out in an ultrahigh vacuum molecular beam scattering machine that contained an independently rotatable crystal manipulator and quadrupole mass spectrometer detector. The arc formed by the detector rotation defines the plane in which scattered atoms can be detected. The Au(111) crystal could be resistively heated and cooled with liquid nitrogen. Cleanliness and order could be checked with Auger electron spectroscopy and He atom beam scattering. All of the data were taken using a postcollision mechanical cross-correlation chopper that was mounted on, and rotated with, the 


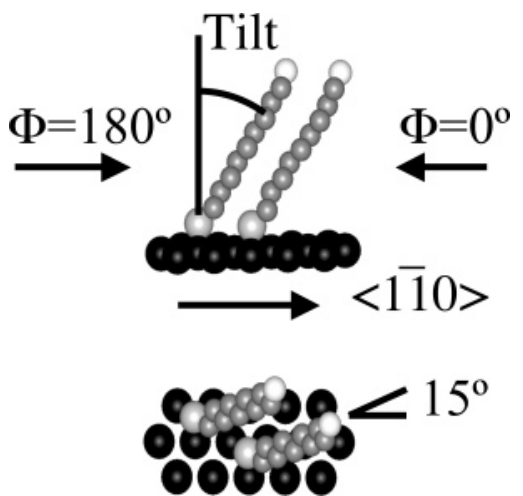

Figure 1. Schematic of the 1-decanethiol/Au surface showing the chain tilt with respect to the Au surface plane ( $34^{\circ}$ experimentally and $38^{\circ}$ for the simulation) and the rotation angle with respect to the $\mathrm{Au}\langle 1 \overline{1} 0\rangle$ direction. In this paper, the azimuthal angle of the incoming $\mathrm{Ar}$ (the angle in the surface plane), $\Phi_{\mathrm{i}}$, is defined as $180^{\circ}$ for scattering along the $\mathrm{Au}\langle 1 \overline{1} 0\rangle$ direction (approximately with the chain tilt) and $0^{\circ}$ in the opposite direction (approximately against the chain tilt).

differentially pumped and collimated detector. Growth of the monolayer was done by exposing the $280 \mathrm{~K} \mathrm{Au}$ crystal to a supersonic beam of He that had been bubbled through 1-decanethiol in a heated reservoir. Molecular beams of Xe were made by the seeded supersonic expansion of a $\mathrm{Xe}-\mathrm{H}_{2}$ mixture (15 psig Xe to 1000 psig $\mathrm{H}_{2}$ ) through a heatable nozzle. The two beams used were $1540 \mathrm{meV}$ (room-temperature nozzle, 100 $\mathrm{meV}$ fwhm) and $3600 \mathrm{meV}$ (773 K nozzle, $450 \mathrm{meV}$ fwhm).

Once an ordered decanethiol monolayer was grown, it was stable for many days if the surface temperature was kept at 200 $\mathrm{K}$ or below, and so a surface was used for many scattering
TABLE 1: Parameters for the $\mathrm{Xe} / \mathrm{CH}_{4}$ Parameters

\begin{tabular}{cll}
\hline parameter & \multicolumn{1}{c}{ value } & \multicolumn{1}{c}{ units } \\
\hline$a$ & $1.592 \times 10^{7}$ & $\mathrm{kcal} /\left(\mathrm{mol} \AA^{12}\right)$ \\
$b$ & -2266 & $\mathrm{kcal} /\left(\mathrm{mol} \AA^{6}\right)$ \\
$c$ & $3.419 \times 10^{5}$ & $\mathrm{kcal} / \mathrm{mol}$ \\
$d$ & 3.3 & $\AA^{-1}$ \\
$f$ & $-3.591 \times 10^{5}$ & $\mathrm{kcal} /\left(\mathrm{mol} \AA^{9}\right)$
\end{tabular}

measurements. He diffraction was used to check the surface ordering during the course of the experiments, and there was no experimentally observable difference in the spectra even after long exposure to hyperthermal $\mathrm{Xe}$, strongly suggesting that there is no collision-induced desorption.

To understand our experiments, we performed calculations similar to those used for our $\mathrm{Ne}$ and Ar results. ${ }^{1-3}$ The purpose was not to evaluate the relative effectiveness of different potentials, but to qualitatively understand the underlying physics. Scattering calculations were done with the VENUS code ${ }^{19}$ using a 36 chain slab, with the addition of periodic boundary conditions to mimic a larger surface without the computational overhead. ${ }^{20}$ As with the $\mathrm{Ne}$ and Ar simulations, a united atom model was used, where the hydrogen and carbon are rigidly connected. The intrachain forces are the same as used in the previous calculations. However, preliminary calculations using the Universal Force Field ${ }^{21,22}$ model for the interchain forces led to what was apparently too much Xe penetration when compared to the experimental results. One way to correct this was to strengthen the interchain forces. Therefore, the $\mathrm{C}-\mathrm{C}$ interchain forces $\left(\mathrm{CH}_{3}-\mathrm{CH}_{3}, \mathrm{CH}_{3}-\mathrm{CH}_{2}\right.$, and $\left.\mathrm{CH}_{2}-\mathrm{CH}_{2}\right)$ were replaced with the results of a $\mathrm{CH}_{4}-\mathrm{CH}_{4}$ potential determined from crossed-beam scattering measurements. ${ }^{23}$ The results,
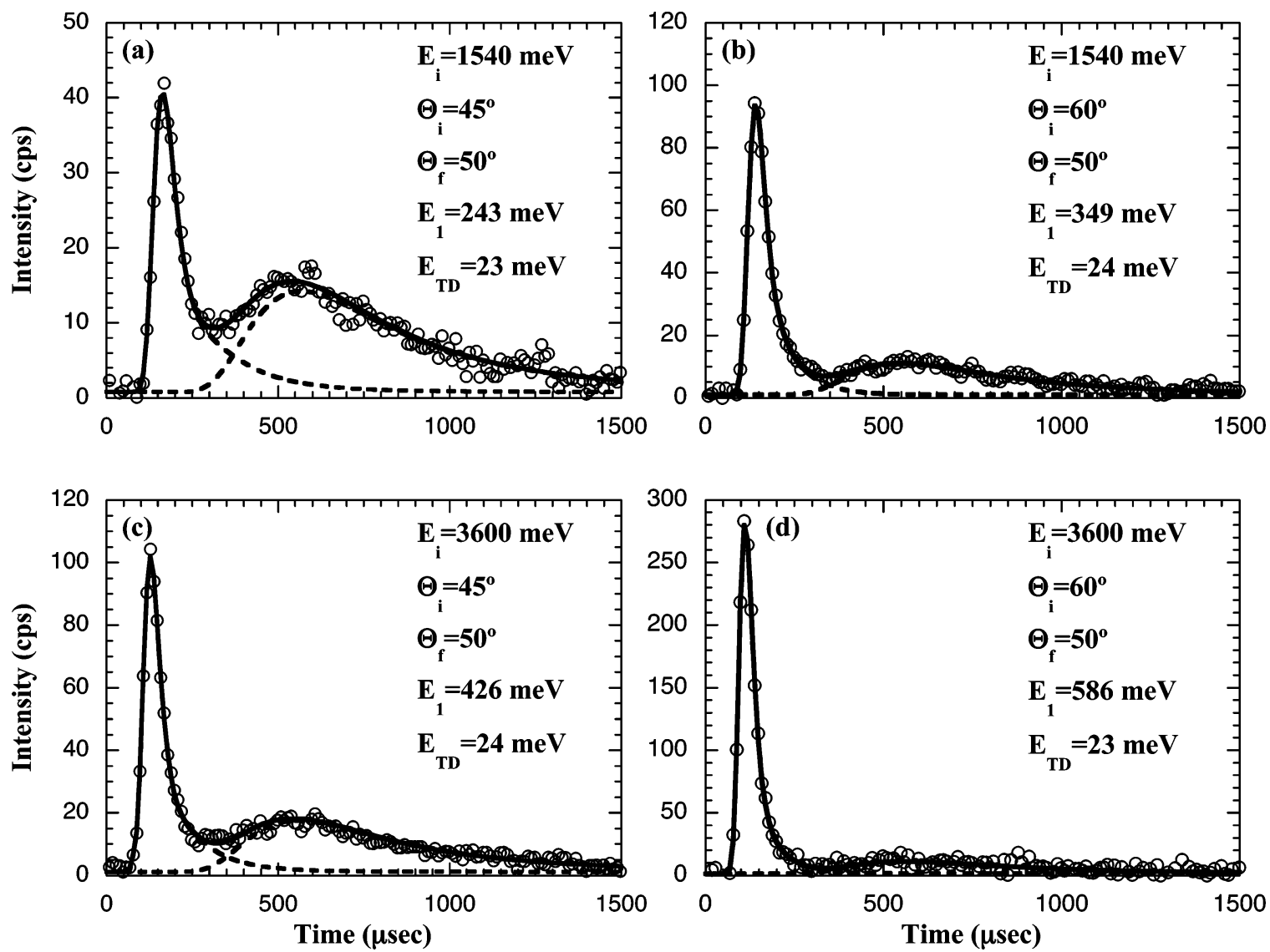

Figure 2. Example TOF spectra, taken at $T_{\mathrm{s}}=135 \mathrm{~K}$ and with the other conditions indicated on each plot. Circles are the data, the solid line is the total fit to the data, and the dashed lines are the individual contributions of the two velocity distributions used in fitting. The average kinetic energy of each distribution is also indicated. 
parametrized as a 6-12 potential, are

$$
V(r)=\frac{8.751 \times 10^{6}}{r^{12}}-\frac{3659}{r^{6}}
$$

where $r$ is in $\AA$ and $V(r)$ in $\mathrm{kcal} / \mathrm{mol}$. This potential has a slightly closer minimum distance and is more than twice as deep as any of the potentials it replaces. The summation of the interactions is done for nearest-neighbor chains, out to a distance of approximately $10 \AA$. Although these new parameters lead to a chain tilt of $38^{\circ}$ at a surface temperature, $T_{\mathrm{s}}$, of $135 \mathrm{~K}$ when the experimental result indicates a tilt angle closer to $34^{\circ},{ }^{24}$ we feel it is a valid model for qualitative comparisons. The orientation of the monolayer is schematically shown in Figure 1. For the $\mathrm{Xe}$ interaction, only the $\mathrm{Xe}-\mathrm{CH}_{3}$ and $-\mathrm{CH}_{2}$ potentials were used for the $\mathrm{Xe}-$ monolayer interaction, derived by fitting the results of $\mathrm{Xe}-\mathrm{CH}_{4}$ crossed-beam scattering experiments ${ }^{25}$ to the equation

$$
V(r)=a / r^{12}+b / r^{6}+c \exp (-d r)+f / r^{9}
$$

The parameters are listed in Table 1.

For a truly quantitative comparison, the united atom model is probably not ideal; at the high velocities and mass of the incoming projectile, it is necessary to consider the highfrequency $\mathrm{C}-\mathrm{H}$ vibrations. ${ }^{26,27}$ Also, at the point of impact, the decanethiol chains can be severely distorted, so that the harmonic potentials used are also poor approximations. Even with the lighter and lower incident energy projectiles, it is apparent from our previous work that the potentials used give good qualitative agreement and clear physical insight. Recent calculations ${ }^{28}$ have shown that there are potentials that more accurately replicate the scattering data than the Universal Force Field ${ }^{21,22}$ and gas-phase rare gas- $\mathrm{CH}_{4}$ potentials.

Trajectories were started by randomly selecting the aiming point within the unit cell. The numerical integration step-size was $2.5 \times 10^{-4}$ ps. Every 200 steps, the positions of all the atoms were saved. This file was used to determine intermediate results during the course of the Xe trajectory. The initial temperature of the monolayer was $135 \mathrm{~K}$ for all of the simulations shown here. During a trajectory, there was no provision for the flow of energy away from the slab, so the monolayer is hotter at the end of the trajectory. However, this does not have much effect on the promptly scattered Xe, and the Xe-surface potential is so deep that this has little effect on the trapped component, whose lifetime is still much longer than the $15-25$ picoseconds of the typical trajectory.

\section{Results and Discussion}

Hyperthermal Xe. Experiment. Figures 2 and 3 show examples of TOF spectra, after deconvoluting out the crosscorrelation pattern. Because of the low count rates that led to rather noisy data, a running three- or five-point average was sometimes done before deconvolution to smooth the data. This was done on a case-by-case basis, though it does not have much effect on average quantities but can broaden features that are narrow in the time domain. Because the detector uses an electron impact ionizer, the intensity shown is a number density. As is typical for many scattering experiments, there appear to be two distinct components in the TOF spectra. To fit the data, the model starts with the sum of velocity distributions:

$$
\begin{aligned}
& f(v)=\sum_{n} a_{n} v^{3} \exp \left(-\left(\left(v-v_{0 n}\right) / \alpha_{n}\right)^{2}\right)+ \\
& a_{3} v^{3} \exp \left(-m v^{2} / 2 k_{\mathrm{B}} T_{\mathrm{s}}\right)
\end{aligned}
$$
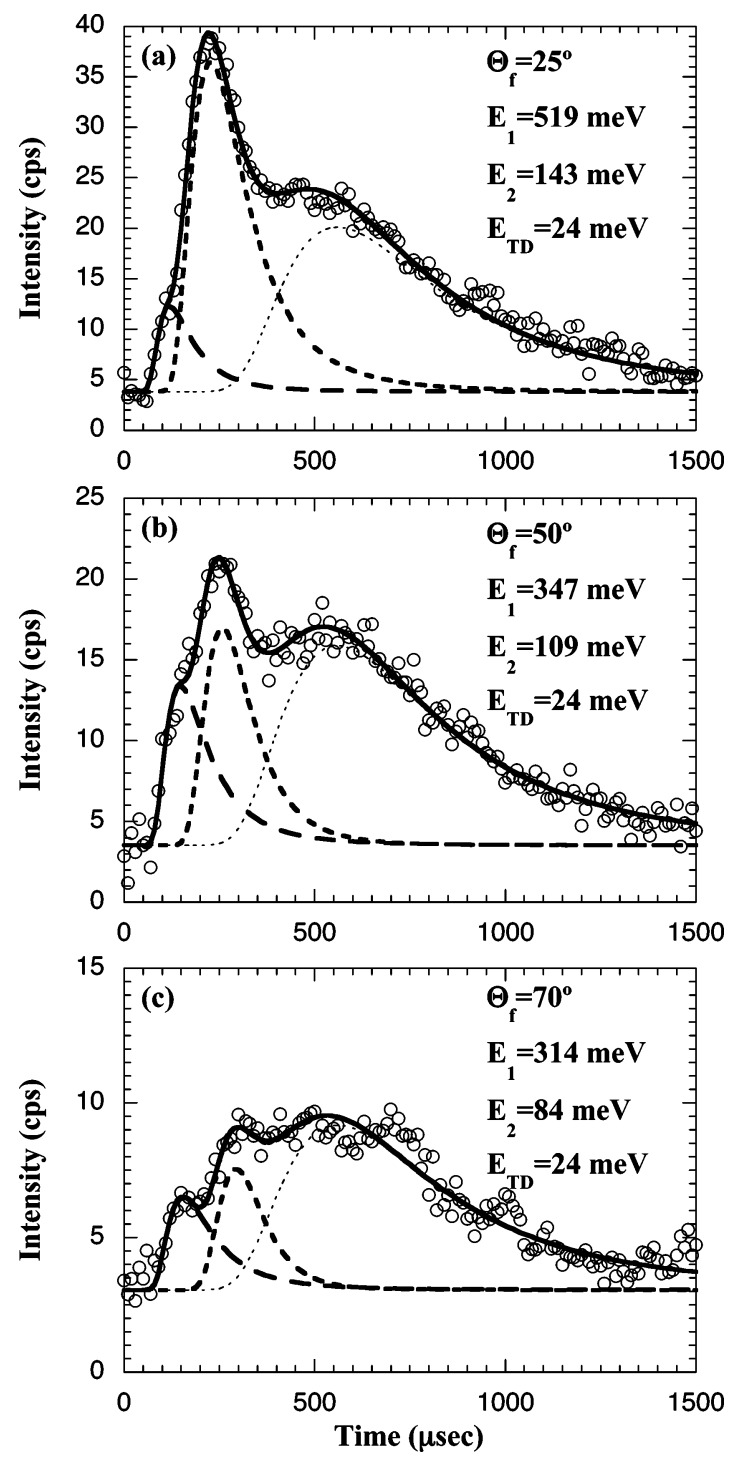

Figure 3. Example TOF spectra with $\Theta_{\mathrm{i}}=20^{\circ}, E_{\mathrm{i}}=3600 \mathrm{meV}$, and $T_{\mathrm{s}}=135 \mathrm{~K}$. The final angle is indicated on the plots. Circles are the data, the solid line is the total fit to the data, and the dashed lines are the individual contributions of each of the three velocity distributions used to fit the data. The average kinetic energy of each distribution is also indicated.

where $n$ is either 1 or 2 , the $a_{n}, v_{0 n}$, and $\alpha_{n}$ are fitting parameters, and $k_{\mathrm{B}}$ is Boltzmann's constant. The first term in the expression represents the sum of shifted Maxwell-Boltzmann distributions. This is used to fit the fast component of the spectra, with the value of $n$ determined by goodness-of-fit. The second term is a Maxwell-Boltzmann distribution at the surface temperature, used to model the slow component in the distribution. The fast term represents the nonthermalized $\mathrm{Xe}$; these atoms were scattered before reaching thermal equilibrium with the surface. The slow term represents Xe that has thermally accommodated with the surface, it has an average energy, $E_{\mathrm{f}}$, of $2 k_{\mathrm{B}} T_{\mathrm{s}}$ and a $\cos \left(\Theta_{\mathrm{f}}\right)$ angular-intensity distribution, where $\Theta_{\mathrm{f}}$ is the exit angle of the scattered Xe.

This equation must be modified to use as a model for the least-squares fitting routine, where eq 3 is used in a forward convolution with the various instrument functions. The first step involves multiplying this equation by the proper Jacobian to convert to a number density. ${ }^{29}$ It must also be corrected for the geometric factors involved in the overlap between the incident beam and the area of the crystal imaged by the well-collimated 

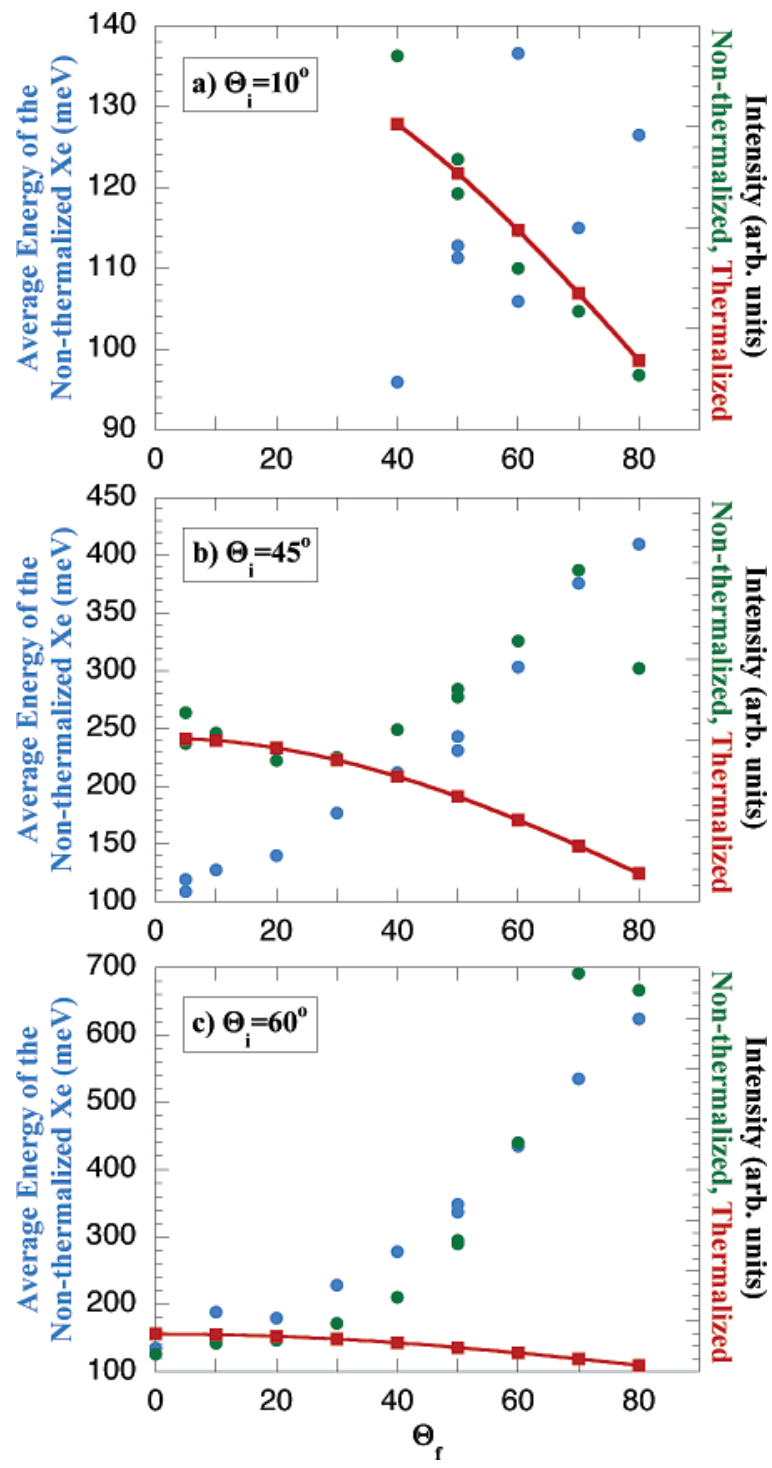

Figure 4. Intensities of the trapped-desorbed Xe (red squares) and the nonthermalized Xe (green circles) and final kinetic energy (blue circles) of the nonthermalized $\mathrm{Xe}$ as a function of final angle from fits such as those shown in Figures 2 and 3. The line through the trappingdesorption component represents a $\cos \left(\Theta_{\mathrm{f}}\right)$ angular-intensity distribution. Multiple points at any final angle are from repeat experiments. Initial conditions are $E_{\mathrm{i}}=1540 \mathrm{meV}$ and $T_{\mathrm{s}}=135 \mathrm{~K}$ at the indicated angle of incidence.

detector ${ }^{30}$ In practice, when $n$ was 1 , the constant $a_{1}$ was sometimes weighted with the sum of the first few Hermite polynomials to improve the fit. ${ }^{31}$ This was done when eq $3 \mathrm{did}$ not fit the data well; the only criterion for whether Hermite polynomials were used and which ones were used was determined by $\chi^{2}$, the goodness-of-fit.

Figures 4 and 5 are summaries of $E_{\mathrm{f}}$ and relative final intensities as a function of $\Theta_{\mathrm{f}}$ for the different incident angles and energies. In all cases, the measured $T_{\mathrm{S}}$ was $135 \mathrm{~K}$, so that $2 k_{\mathrm{B}} T_{\mathrm{s}}=23.3 \mathrm{meV}$. If $T_{\mathrm{s}}$ was allowed to vary when fitting the trapping-desorption component, the fit always gave a result within a few degrees of this temperature. The line is the cos$\left(\Theta_{\mathrm{F}}\right)$ angular-intensity distribution expected for trappingdesorption.

At glancing angles of incidence, the intensity and energy distributions of the nonthermalized component of the Xe spectra peak at large final angles. Similar behavior was seen for $\mathrm{Ne}$ and $\mathrm{Ar}$, as well as high-energy Xe scattering from liquid and
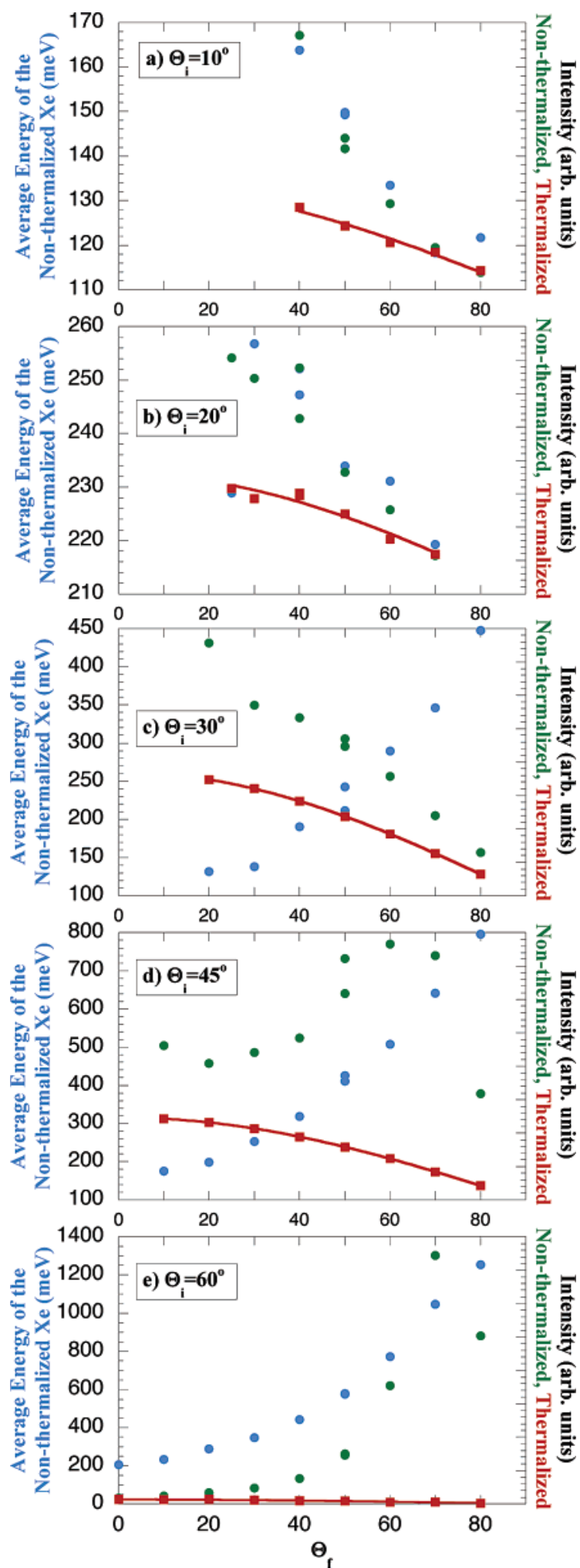

Figure 5. Intensities of the trapped-desorbed $\mathrm{Xe}$ (red squares) and the nonthermalized Xe (green circles) and final kinetic energy (blue circles) of the nonthermalized $\mathrm{Xe}$ as a function of final angle from fits such as those shown in Figures 2 and 3. The line through the trappingdesorption component represents a $\cos \left(\Theta_{\mathrm{f}}\right)$ angular-intensity distribution. Multiple points at any final angle are from repeat experiments. Initial conditions are $E_{\mathrm{i}}=3600 \mathrm{meV}$ and $T_{\mathrm{s}}=135 \mathrm{~K}$ at the indicated angle of incidence. The important point to note is the progression of the exit angle dependence of the energy and intensity of the nonthermalized Xe as a function of incident angle. At glancing angles of incidence, the final energy and intensity peak at glancing final angles. However, at incident angles nearer normal, the final energy and intensity is greatest near more normal exit angles. 

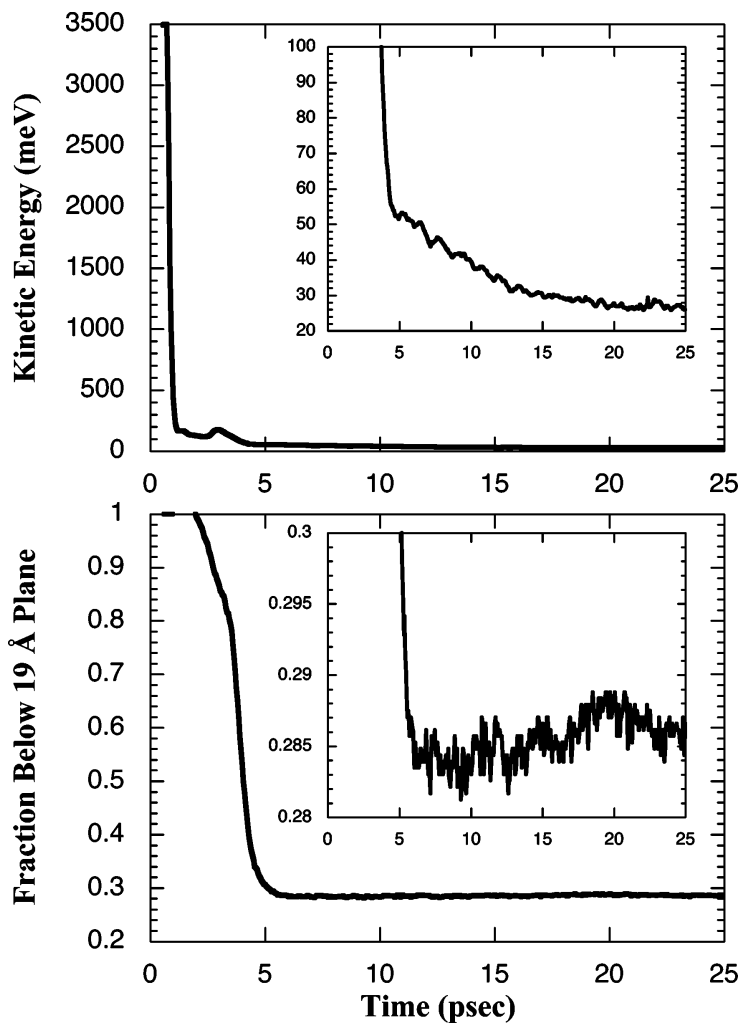

Figure 6. Number of Xe atoms below an imaginary plane $19 \AA$ above the $\mathrm{Au}$ surface and their average kinetic energy as a function of time for 2247 trajectories. Time $=0$ is the start of the trajectories, and the insets show the same data with rescaled $y$-axes. Conditions were $E_{\mathrm{i}}=$ $3500 \mathrm{meV}, \Theta_{\mathrm{I}}=20^{\circ}, \Phi_{\mathrm{I}}=180^{\circ}$, and $T_{\mathrm{s}}=135 \mathrm{~K}$.

semiconductor surfaces. This direct-inelastically scattered component is qualitatively explained by models that consider one, or a few, collisions with surface constituents. However, as the incident angle is varied toward normal, first the intensity, and then the energy, of the fast Xe peaks toward more normal angles. This progression is quite noticeable in Figure 5. With Ar, we saw the energy and intensity of the scattered component peaking near normal with small incident angles, but unlike the Xe, there was not a distinct high-energy peak as in Figure 3.

Though less clear experimentally, there is one other feature of the spectra worth mentioning. For most rare gas scattering, there are, at most, two distinct velocity distributions in the TOF spectra, one due to direct-inelastic scattering and one due to trapping-desorption, or in the case of $\mathrm{Ne}$ from SAMs, near surface accommodation of the impinging atom. Though not clearly evident in all of the Xe TOF spectra, some, such as those shown in Figure 3, show evidence of two distinct features besides that due to trapping-desorption, suggesting that there is more than one channel that leads to scattering without energy accommodation.

Simulations. The simulation uses a simplified surface with a $(\sqrt{3} \times \sqrt{3}) \mathrm{R} 30^{\circ}$ surface structure with a lattice constant of 5 $\AA$. The first step was to determine the time necessary to track a trajectory. Figure 6 shows the fraction of trajectories below a plane $19 \AA$ above the Au surface and their average kinetic energy. (All surface normal distances are given with respect to the plane of the static Au crystal.) It is clear that the Xe either leaves the surface very promptly or is rapidly equilibrated with it. In consequence, the calculations were truncated at times between 15 and 25 ps. Most of the calculations were performed with a 25 ps cutoff, to ensure that the Xe had equilibrated with the surface; at shorter times there could be a few trajectories
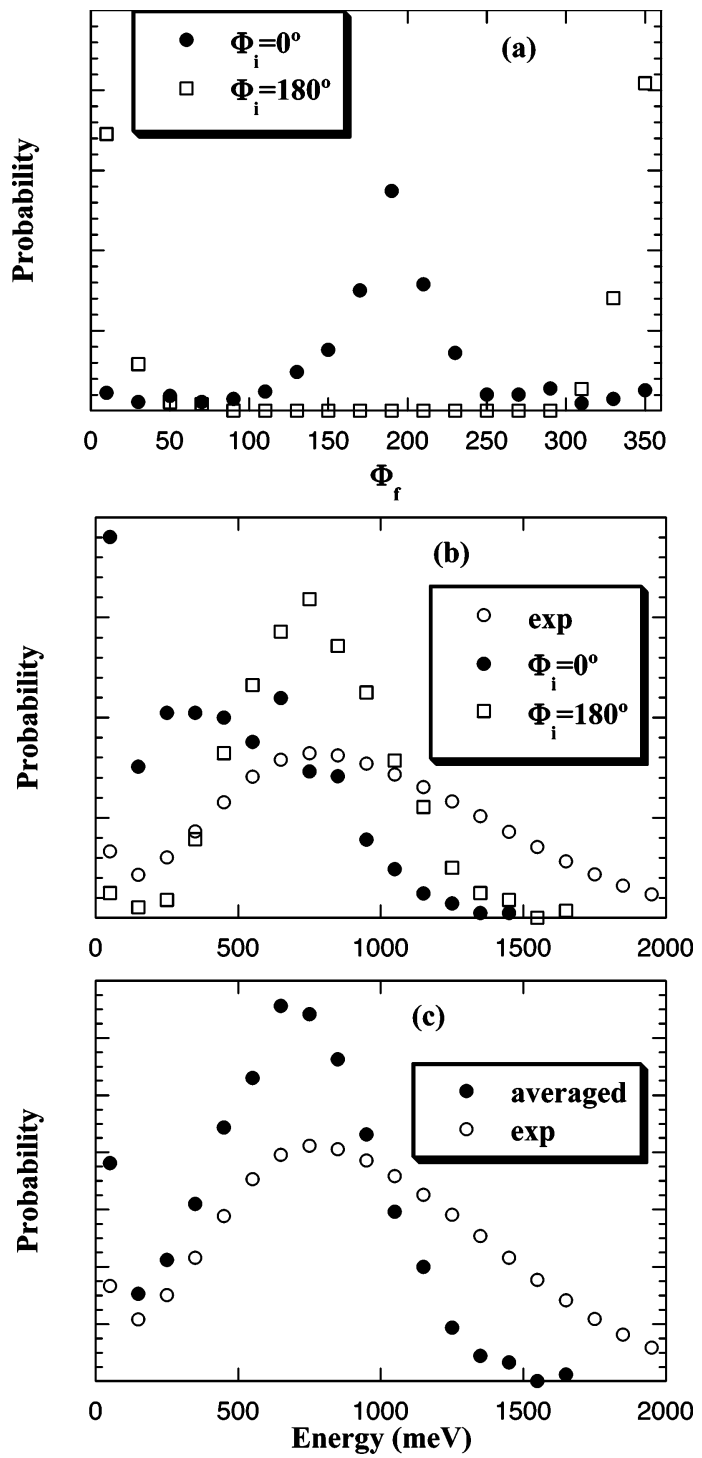

Figure 7. Comparison of experimental and theoretical kinetic energy distributions for all $\Theta_{\mathrm{f}}$, with $E_{\mathrm{i}}=3600 \mathrm{meV}, \Theta_{\mathrm{i}}=60^{\circ}$, and $T_{\mathrm{s}}=135$ K. Figure 7 a shows the azimuthal angular dependence of the scattered Xe. Figure $7 \mathrm{~b}$ shows the sum of scattered and trapped-desorbed components for the simulations with $\Phi_{\mathrm{i}}=0^{\circ}\left(\Phi_{\mathrm{f}}=180 \pm 40^{\circ}\right)$ and $\Phi_{\mathrm{i}}=180^{\circ}\left(\Phi_{\mathrm{f}}=0 \pm 40^{\circ}\right)$. Figure $7 \mathrm{c}$ is their weighted sum.

that had not equilibrated. Any Xe remaining on the surface was assumed to be trapped, and to desorb as a Maxwell-Boltzmann velocity distribution with a temperature of $135 \mathrm{~K}$ and a cosine angular-intensity distribution.

Figure 7 shows a comparison between the experiment and simulation for the glancing incident angle of $\Theta_{\mathrm{i}}=60^{\circ}$. The simulations were done at two different incident azimuths, $0^{\circ}$ and $180^{\circ}$. It was impossible to run enough trajectories for a direct comparison with the experiment (detector resolution $\sim 1^{\circ}$ fwhm) so a wider range of azimuths must be used, as detailed in the captions. This makes the comparisons qualitative, because at this angle of incidence, the directly scattered Xe comes off in a sharply peaked azimuthal angular range. As shown in Figure 7a, the fast molecules are always forward scattered; if $\Phi_{\mathrm{i}}=0^{\circ}$, $\Phi_{\mathrm{f}}=180^{\circ}$, and vice versa. As is clear in Figure $7 \mathrm{~b}$, there is a dependence of both the energy and proportion of the directly scattered $\mathrm{Xe}$ on the incident azimuthal angle. The experimental SAM surface is made up of a mosaic of rotated domains, and even domains that are aligned can have the tilt direction vary by $180^{\circ}$ and the azimuthal angle be $\pm 15^{\circ}$ with respect to this 

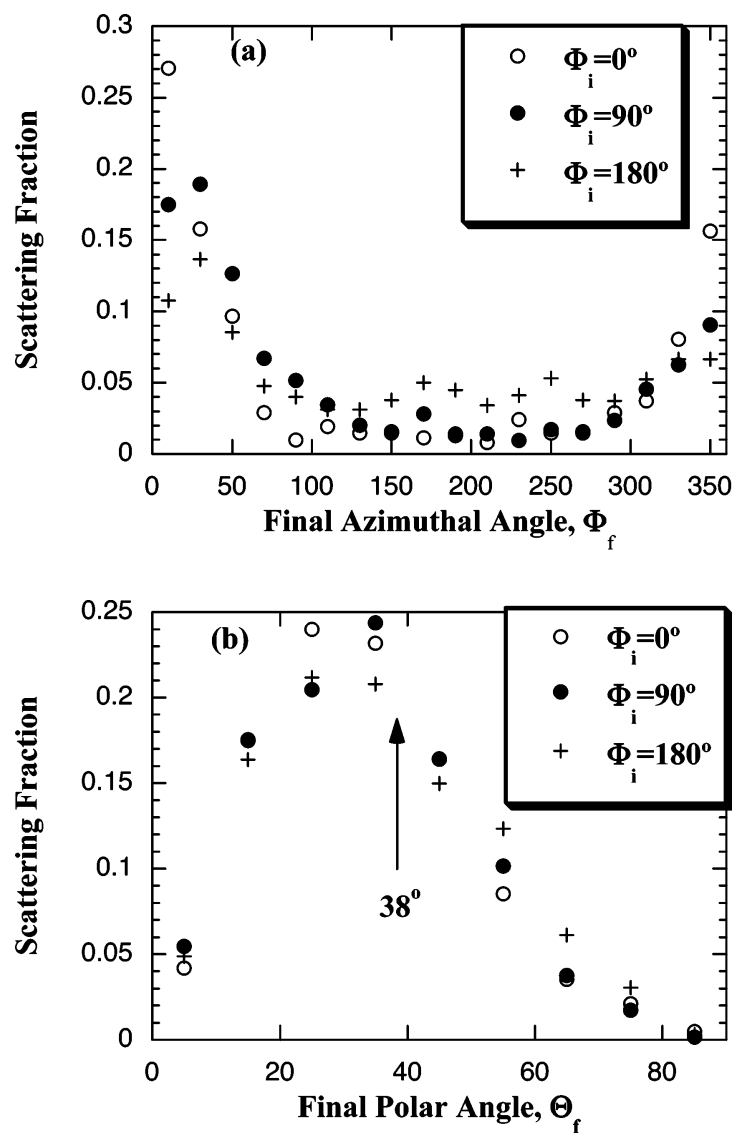

Figure 8. Final polar and azimuthal angle dependence of the atoms that escaped the surface within $25 \mathrm{ps}$ at three different $\Phi_{\mathrm{i}}$. Incident conditions were $E_{\mathrm{i}}=3500 \mathrm{meV}, \Theta_{\mathrm{i}}=20^{\circ}$, and $T_{\mathrm{s}}=135 \mathrm{~K}$. The tilt angle of the simulation is $38^{\circ}$.

tilt. For comparison, just the azimuthal angles of $0^{\circ}$ and $180^{\circ}$ were averaged. The result is shown in Figure 7c, with the distributions normalized to unity. Estimated error bars are slightly larger than the symbols, so the divergence is real, with the simulation overestimating the amount of energy exchange. However, the two distributions are qualitatively the same, with the high-energy component having a peak at approximately 800 $\mathrm{meV}$. Analogously with other experiments, this is assigned to a direct-inelastic component. The closest distance to the $\mathrm{Au}$ surface is almost always greater than $12 \AA$ for the $\mathrm{Xe}$ trajectories, where the average position of the $\mathrm{CH}_{3}$ terminal group is approximately $11.9 \AA$. The colliding Xe deforms the chains near the point of collision but still scatters off the surface of the monolayer.

Figure 8 shows the angular intensity for an incident angle much closer to normal: Xe with an initial energy of $3500 \mathrm{meV}$, $\Theta_{i}=20^{\circ}$, and three different incident azimuthal angles. Figure 8 a shows the final azimuthal angle. Though the distributions are not identical, at all of the incident azimuths the peak is at

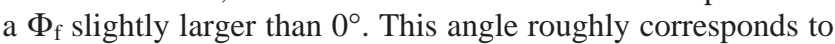
the azimuthal rotation of the chains, $15^{\circ}$. Figure $8 \mathrm{~b}$ shows the polar angle dependence. These distributions all peak at 25$35^{\circ}$, quite near the tilt angle of the chains (see Figure 1). In summary, no matter what the incident azimuth, the Xe atoms that are nonthermalized leave the surface directed along the direction of the 1-decanethiol chains.

Figure 9 shows a comparison between the experiment and the simulation at $\Theta_{\mathrm{i}}=20^{\circ}$ and $E_{\mathrm{i}}=3500 \mathrm{meV}$ (simulation) and $E_{\mathrm{i}}=3600 \mathrm{meV}$ (experiment). Examining Figure 8a, in the experiment any domain that had the tilt oriented toward the incident beam had the nonthermalized Xe leave the surface in

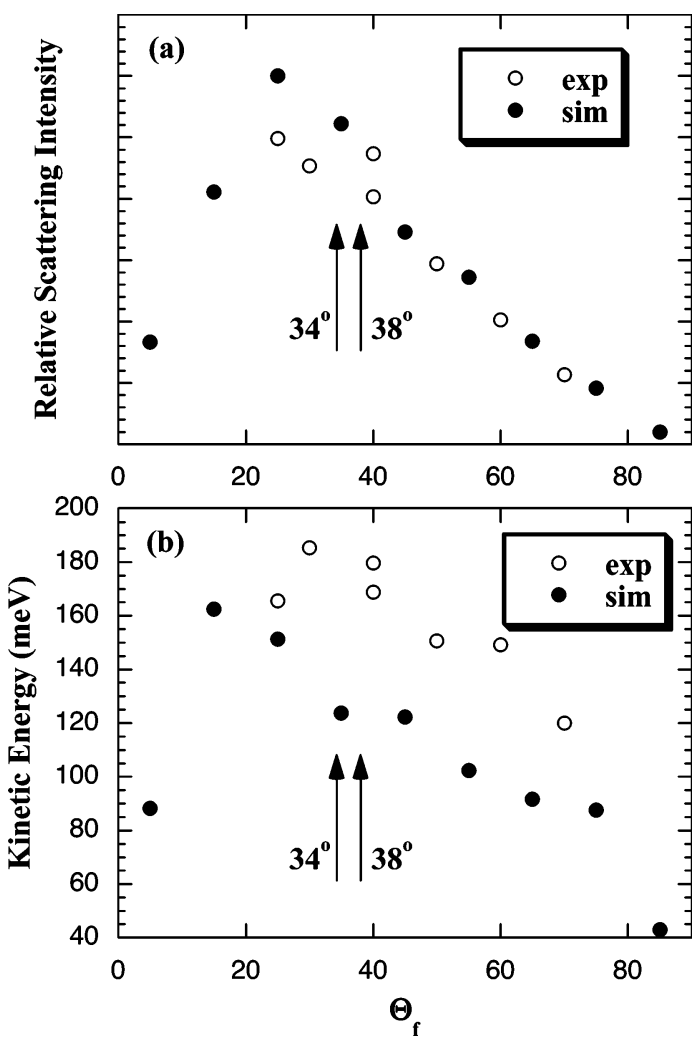

Figure 9. Comparison of the total (including the trapped-desorbed) kinetic energy and intensity as a function of $\Theta_{\mathrm{f}}$ for the experiment $\left(E_{\mathrm{i}}\right.$ $=3600 \mathrm{meV})$ and simulation $\left(E_{\mathrm{i}}=3500 \mathrm{meV}, \Phi_{\mathrm{i}}=180^{\circ}\right.$, and $\Phi_{\mathrm{f}}=$ $0 \pm 50^{\circ}$ ) with $\Theta_{\mathrm{i}}=20^{\circ}$ and $T_{\mathrm{s}}=135 \mathrm{~K}$. The tilt angles are $38^{\circ}$ for the simulation and $34^{\circ}$ for the experimental surface.

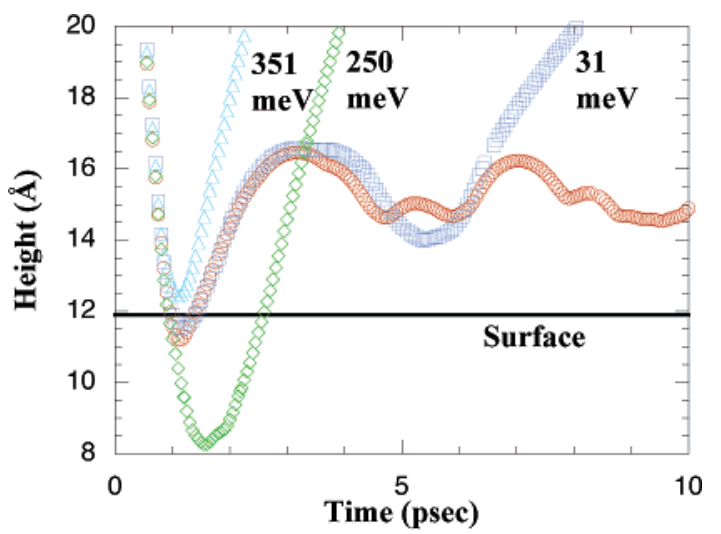

Figure 10. Examples of four trajectories for $E_{\mathrm{i}}=3500 \mathrm{meV}, \Theta_{\mathrm{i}}=$ $20^{\circ}, T_{\mathrm{s}}=135 \mathrm{~K}$, and $\Phi_{\mathrm{i}}=180^{\circ}$. The three that left the surface are labeled with their final energy, the other was still trapped at 25 ps. The terminal $\mathrm{CH}_{3}$ is at $11.9 \AA$ in the simulation.

a direction inaccessible to the detector. Any domain that was oriented with the tilt at $90^{\circ}$ to the direction of the beam had the Xe leave the surface mostly out of plane. With these considerations, the main contribution will be from domains where the tilt is oriented away from the incident direction of the beam. Therefore, the comparison is done with only the $\Phi_{\mathrm{i}}=180^{\circ}$ simulation. Qualitatively, the agreement is quite good, with the angular dependence of both the energy and intensity increasing toward a more normal final polar angle.

Some trajectories are plotted in Figure 10. Two observations are clear: the Xe penetrates quite deeply into the monolayer but are not trapped there for very long, and can still leave the surface with a considerable amount of energy. In Figure 11 are shown the minimum $z$-distance distribution for trajectories run 


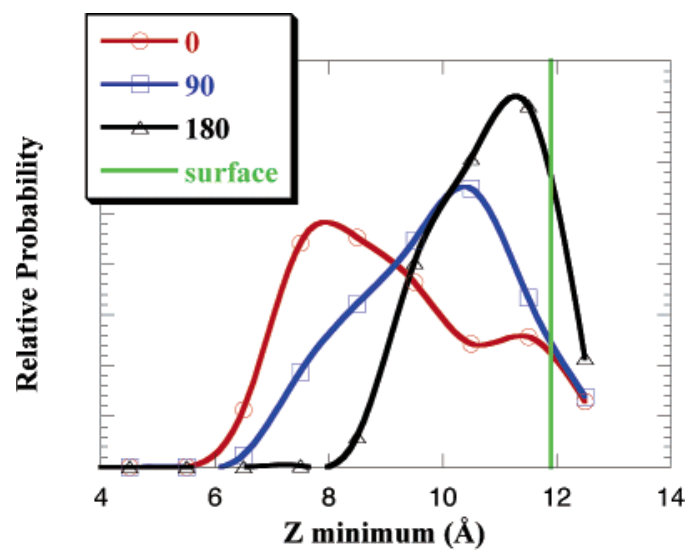

Figure 11. Comparison of the distributions of the minimum $z$ distance for all of the trajectories at three different incident azimuthal angles. Conditions are $E_{\mathrm{i}}=3500 \mathrm{meV}, \Theta_{\mathrm{i}}=20^{\circ}$, and $T_{\mathrm{s}}=135 \mathrm{~K}$. Lines are just a guide for the eye.
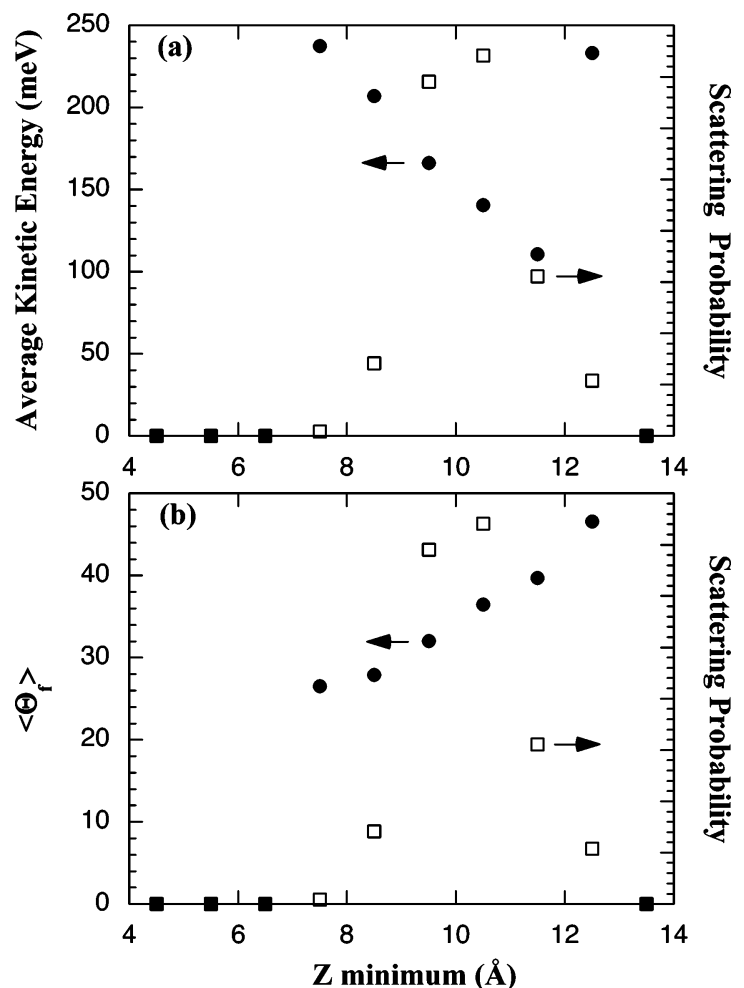

Figure 12. Average final kinetic energy and average final polar angle (left axes, solid circles) as a function of the minimum $z$ distance for trajectories that escaped the surface within 25 ps compared with the minimum $z$ distribution (right axes, open squares). Conditions are $E_{\mathrm{i}}$ $=3500 \mathrm{meV}, \Theta_{\mathrm{i}}=20^{\circ}, T_{\mathrm{s}}=135 \mathrm{~K}$, and $\Phi_{\mathrm{i}}=180^{\circ}$.

at $E_{\mathrm{i}}=3500 \mathrm{meV}, \Theta_{\mathrm{i}}=20^{\circ}$, and $T_{\mathrm{s}}=135 \mathrm{~K}$ at three different incident azimuths. On average, the deepest penetration is reached when the incident direction is approximately along the tilt direction, $\Phi_{\mathrm{i}}=0^{\circ}$. However, at all angles the average penetration is far into the monolayer.

Figure 12 shows the minimum $z$ distribution for just the trajectories that left the surface within $25 \mathrm{ps}$ with $\Phi_{\mathrm{i}}=180^{\circ}$ and $\Phi_{\mathrm{f}}=0 \pm 50^{\circ}$, along with the dependence of the average final kinetic energy and polar angle on the minimum $z$ reached by the Xe. Comparing Figures 11 and 12, it is apparent that, for this particular azimuth, the most energetic Xe leaving the surface on average penetrates deeper. The plots in Figure 12 also suggest a reason for the appearance of two fast features in some of the TOF spectra. The final average kinetic energy increases as the Xe penetrates further into the monolayer, except

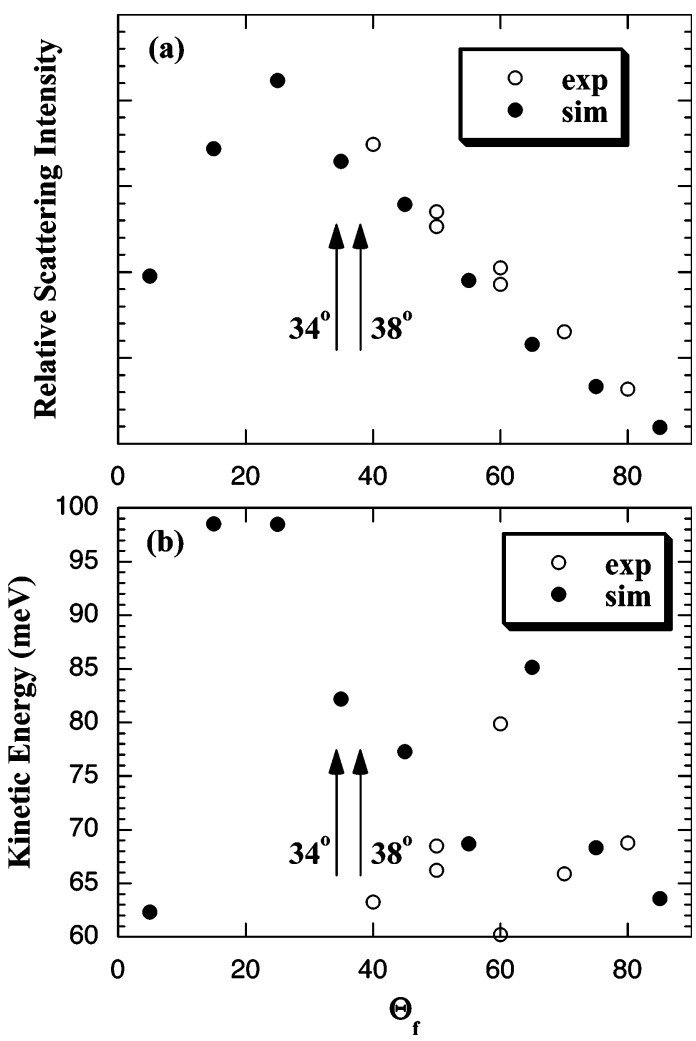

Figure 13. Comparison of total kinetic energy and intensity as a function of $\Theta_{\mathrm{f}}$ for the experiment and simulation $\left(\Phi_{\mathrm{i}}=180^{\circ}\right.$ and $\Phi_{\mathrm{f}}$ $=340-100^{\circ}$ ) for $\Theta_{\mathrm{i}}=10^{\circ}, E_{\mathrm{i}}=1540 \mathrm{meV}$, and $T_{\mathrm{s}}=135 \mathrm{~K}$.

for the trajectories that scatter without penetrating very far. The former are squeezed out of the monolayer as the chains try to return to their equilibrium position, the directed ejection, whereas the latter are due to direct-inelastic scattering from the surface. Examples of these two types of trajectories are shown in Figure 10, where one of the trajectories that leaves the monolayer promptly deeply penetrates and the other scatters from the surface. This contention is supported by the average final angle as a function of $z$ minimum. If it penetrates deeper, Xe leaves the monolayer more toward the direction of the chain tilt, whereas the direct-inelastic component comes off at more glancing angles.

Mass and Energy Dependence. The mass and energy (velocity) dependence of the penetration was also briefly investigated. Figure 13 shows plots similar to those in Figure 9 for $E_{\mathrm{i}}=1540 \mathrm{meV}$ and $\Theta_{\mathrm{i}}=10^{\circ}$. Though not shown, the simulation indicated an azimuthal and polar angle dependence of the scattering is similar to that for $E_{\mathrm{i}}=3500 \mathrm{meV}$ and $\Theta_{\mathrm{i}}=$ $20^{\circ}$.

Experiments were also done with Ar with $E_{\mathrm{i}}=2100 \mathrm{meV}$ (fwhm $=280 \mathrm{meV}$ ) and $\Theta_{\mathrm{i}}=20^{\circ}$ at $T_{\mathrm{s}}=135 \mathrm{~K}$. Some of the TOF spectra are shown in Figure 14, and the comparison between the experiment and the theory is shown in Figure 15. The intensity dependence on the final azimuthal angle is not as peaked as in Figure 8, but when $\Phi_{i}=0^{\circ}$, the nonthermalized $\mathrm{Xe}$ is still largely directed away from the detector, so the comparison is only done with $\Phi_{\mathrm{i}}=180^{\circ}$. The intensities are similar, and both the experiment and simulation show an increase in final average kinetic energy at glancing angles, though the experimental change is much larger. However, the simulation qualitatively shows the same trend as the experiments, with the hyperthermal Xe showing an increase in final energy toward normal final angles and the Ar showing an increase in average energy toward glancing angles. 

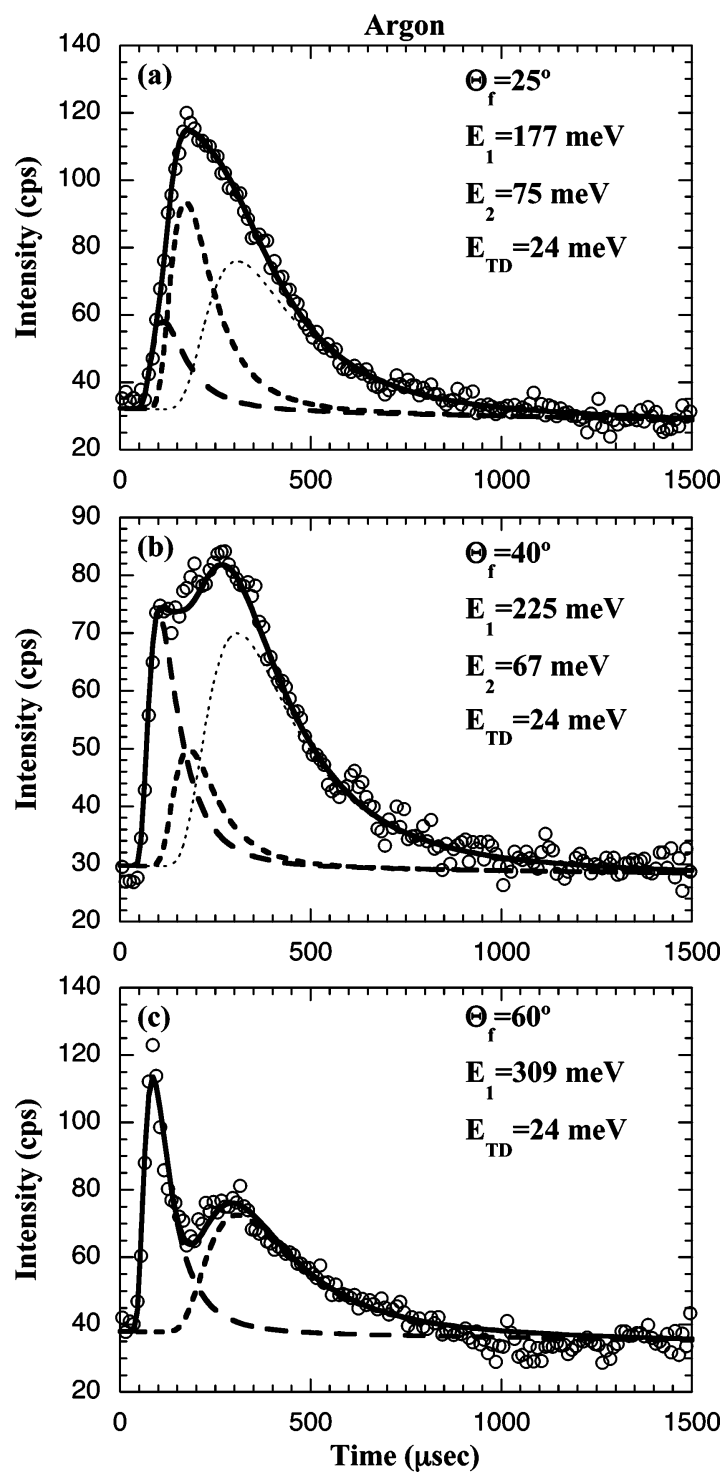

Figure 14. Examples of Ar TOF spectra with $\Theta_{\mathrm{i}}=20^{\circ}, E_{\mathrm{i}}=2100$ $\mathrm{meV}$, and $T_{\mathrm{s}}=135 \mathrm{~K}$. Final angle is indicated on the plots. Circles are the data, the solid line is the total fit to the data, and the dashed lines are the individual contributions of each of the velocity distributions used to fit the data. The average kinetic energy of each distribution is also indicated.

Figure 16 compares the minimum $z$ distributions for the Xe and Ar colliding with the surface at near normal angles. Although much of the Xe penetrates, much of the Ar has a minimum $z$ near or above the equilibrium position of the terminal methyl group. The much less massive Ar atoms are unable to push aside the chains and penetrate the surface, so most of the impinging $\mathrm{Ar}$ is inelastically scattered, with the final energy increasing toward more glancing final angles; at these energies the directed ejection channel is closed for Ar.

\section{Conclusions}

In this paper, we have discussed experiments for hyperthermal Xe colliding with an ordered 1-decanethiol surface. Experimentally, there are both fast components and accommodated components in the time-of-flight spectra. The latter, the trappeddesorbed component, has an average energy of $2 k_{\mathrm{B}} T_{\mathrm{s}}$, and a $\cos \left(\Theta_{\mathrm{f}}\right)$ angular-intensity distribution. At glancing angles of incidence, the fast component shows an increase in intensity and average kinetic energy at glancing final angles. This is what
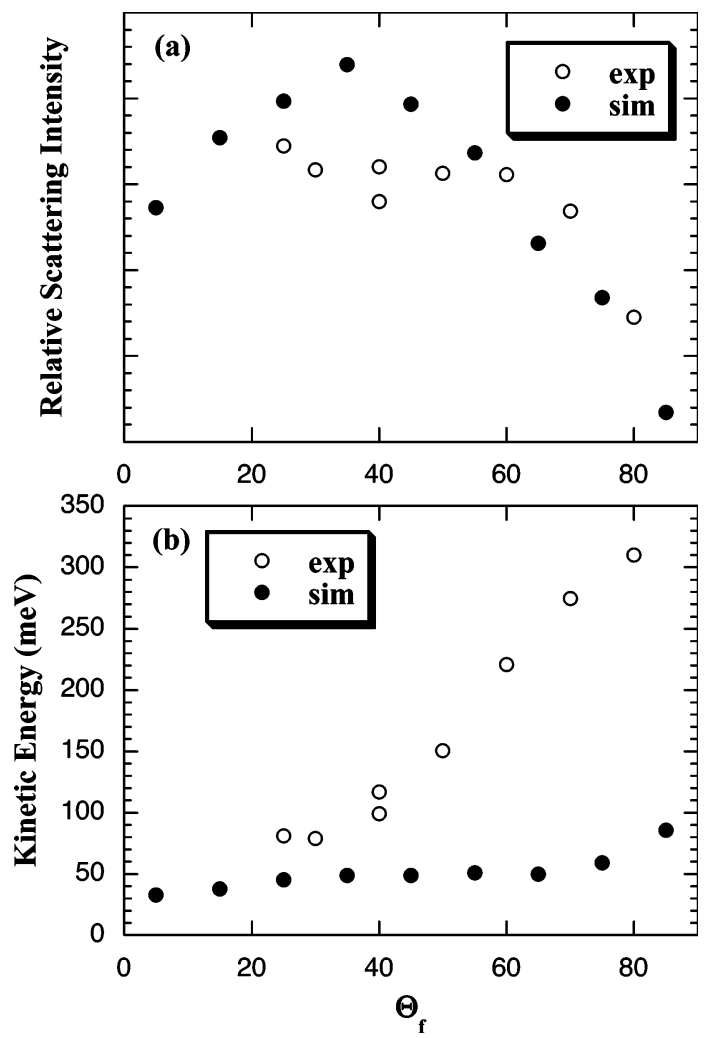

Figure 15. Comparison of total kinetic energy and intensity of scattered Ar as a function of $\Theta_{\mathrm{f}}$ for the experiment and simulation $\left(\Phi_{\mathrm{i}}=180^{\circ}\right.$ and $\left.\Phi_{\mathrm{f}}=340-100^{\circ}\right)$, with $\Theta_{\mathrm{i}}=20^{\circ}, E_{\mathrm{i}}=2100 \mathrm{meV}$, and $T_{\mathrm{s}}=135$ $\mathrm{K}$.

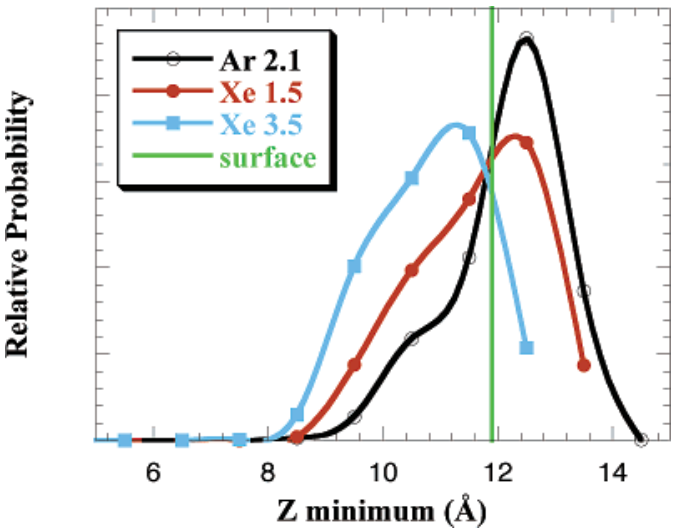

Figure 16. Comparison of the distributions of the minimum $z$ distance for all of the trajectories for $\mathrm{Xe}\left(E_{\mathrm{i}}=3500 \mathrm{meV}, \Theta_{\mathrm{i}}=20^{\circ}\right), \mathrm{Xe}\left(E_{\mathrm{i}}=\right.$ $\left.1540 \mathrm{meV}, \Theta_{\mathrm{i}}=10^{\circ}\right)$, and $\operatorname{Ar}\left(E_{\mathrm{i}}=2100 \mathrm{meV}, \Theta_{\mathrm{i}}=20^{\circ}\right)$, all with $\Phi_{\mathrm{i}}$ $=180^{\circ}$ and $T_{\mathrm{s}}=135 \mathrm{~K}$. Lines are just a guide for the eye.

is expected for direct-inelastic scattering. At more normal $\Theta_{\mathrm{i}}$, the nature of this fast component changes, with the energy and intensity increasing toward more normal final angles.

To understand this transition, we undertook scattering calculations. The qualitative agreement with the experimental results give clear physical insight, indicating a third scattering mechanism. This involves the penetration of the Xe into the interstitial spaces between the neighboring chains, which are displaced. The incident energy is quickly dissipated, and the $\mathrm{Xe}$ is pushed out of the interior of the SAM as the chains return to their equilibrium positions. The $\mathrm{Xe}$ is expelled from the surface at an energy well above thermal, and with the intensity peaked toward the average orientation of the 1-decanethiol chains. We term this new scattering channel directed ejection. 
Acknowledgment. We thank Tianying Yan and William L. Hase of Wayne State University for helpful discussions and supplying us the VENUS code with the necessary modifications for the simulations. This work was primarily supported by the AFOSR sponsored MURI Center for Materials Chemistry in the Space Environment. Supplemental support from the National Science Foundation-Materials Research Science and Engineering Center at the University of Chicago is also gratefully acknowledged. We also acknowledge the Burroughs Wellcome Fund Interfaces Cross-Disciplinary Training Program at the University of Chicago for partial support of the computer cluster.

\section{References and Notes}

(1) Isa, N.; Gibson, K. D.; Yan, T.; Hase, W. L.; Sibener, S. J. J. Chem. Phys. 2004, 120, 2417.

(2) Yan, T.; Hase, W. L.; Isa, N.; Gibson, K. D.; Sibener, S. J. J. Phys. Chem. A 2003, 107, 10600 13083.

(3) Gibson, K. D.; Isa, N.; Sibener, S. J. J. Chem. Phys. 2003, 119,

(4) Ellison, M. D.; Matthews, C. M.; Zare, R. N. J. Chem. Phys. 2000, $112,1975$.

(5) Rettner, C. T.; Barker, J. A.; Bethune, D. S. Phys. Rev. Lett. 1991, 67, 2183.

(6) Barker, J. A.; Rettner, C. T. J. Chem. Phys. 1992, 97, 5844.

(7) Barker, J. A.; Rettner, C. T. J. Chem. Phys. 1994, 101, 9202

(8) Amirav, A.; Cardillo, M. J.; Trevor, P. L.; Lim, C.; Tully, J. C. J. Chem. Phys. 1987, 87, 1796

(9) Lim, C.; Tully, J. C.; Amirav, A.; Trevor, P. L.; Cardillo, M. J. J. Chem. Phys. 1987, 87, 1808.

(10) King, M. E.; Nathanson, G. M. Phys. Rev. Lett. 1993, 70, 1026.

(11) Lipkin, N.; Gerber, R. B.; Moiseyev, N.; Nathanson, G. M. J. Chem. Phys. 1994, 100, 8408.
(12) Ronk, W. R.; Kowalski, D. V.; Manning, M.; Nathanson, G. M. J. Chem. Phys. 1996, 104, 4842.

(13) Bolton, K.; Pettersson, J. B. C. Chem. Phys. Lett. 1999, 312, 71.

(14) Day, B. S.; Morris, J. R. J. Chem. Phys. 2005, 122.

(15) Day, B. S.; Morris, J. R.; Alexander, W. A.; Troya, D. J. Phys. Chem. A, in press.

(16) Sibener, S. J. Proc. Symp. At., Surf., Cluster Phys., IV 2004.

(17) Sibener, S. J.; Cisse, A.; Darling, S. B.; Freedman, M.; Gibson, K. D.; Lee, D.-C.; Rosenbaum, A. W.; Wang, Y.; Yu, L. Abstracts of Papers; 230th ACS National Meeting; American Chemical Society: Washington, DC, 2005.

(18) Colonell, J. I.; Gibson, K. D.; Sibener, S. J. J. Chem. Phys. 1995, $103,6677$.

(19) Hase, W. L.; Duchovic, R. J.; Hu, X.; Kormonicki, A.; K., L.; Lu, D.-H.; Peslherbe, G. H.; Swamy, K. N.; Vande Linds, S. R.; Varandos, A. J. C.; Wang, H.; Wolfe, R. J. QCPE 1996, 16, 671.

(20) Haile, J. M. Molecular Dynamics Simulation: Elementary Methods; John Wiley \& Sons: New York, 1992.

(21) Bosio, S. B. M.; Hase, W. L. J. Chem. Phys. 1997, 107, 9677.

(22) Rappe, A. M.; Casewit, C. J.; Colwell, K. S.; Goddard, W. A., III; Skiff, W. M. J. Am. Chem. Soc. 1992, 114, 10024.

(23) Reid, B. P.; O’Loughlin, M. J.; Sparks, R. K. J. Chem. Phys. 1985, 83,5656 .

(24) Fenter, P. Thin Films 1998, 24, 111.

(25) Liuti, G.; Pirani, F.; Buck, U.; Schmidt, B. Chem. Phys. 1988, 126,

(26) Yan, T.; Hase, W. L. J. Phys. Chem. A 2001, 105, 2617.

(27) Martens, C. C. Chem. Phys. 2002, 280, 257.

(28) Day, B. S.; Morris, J. R.; Troya, D. J. Chem. Phys. 2005, 122.

(29) Auerbach, D. J. Velocity Measurements by Time-of-Flight Methods. In Atomic and Molecular Beam Methods; Scoles, G., Ed.; Oxford University Press: New York, 1988; Vol. 1, p 362.

(30) Comsa, G.; David, R. Surf. Sci. Rep. 1985, 5, 145.

(31) Beijerinck, H. C. W.; Kaashoek, G. H.; Beijers, J. P. M.; Verheijen, M. J. Physica $B \& C \mathbf{1 9 8 3}, 121 B+C, 425$. 